\title{
Pediatric spinal cord infarction following a minor trauma: a case report
}

\author{
Ali Morshid $\mathbb{D}^{1} \cdot$ Huda Al Jadiry ${ }^{1} \cdot$ Umar Chaudhry $^{1} \cdot$ Karthikram Raghuram $^{1}$
}

Received: 26 July 2020 / Revised: 14 September 2020 / Accepted: 18 September 2020

(c) International Spinal Cord Society 2020

\begin{abstract}
Introduction Pediatric spinal cord infarction is a rare entity that presents a diagnostic challenge at the emergency department. Ischemic spinal cord infarction can occur in the setting of trauma, cardiovascular malformation, or postoperatively. We report a case of anterior spinal artery infarction following seemingly minor trauma in an otherwise healthy 14-yearold male.

Case presentation A 14-year-old male presented with unprovoked sudden-onset stabbing back pain earlier that day. The patient then demonstrated bilateral lower extremities weakness while at the emergency department. After extensive diagnostic workup, the patient was diagnosed with anterior spinal artery territory infarction involving the thoracic spinal cord. Minor trauma to the thoracic spinal cord was detected on imaging and was believed to be the culprit of this event.

Discussion Given the rarity of spinal cord ischemia, a high index of suspicion and extensive workup of patients presenting with clinical weakness are needed for accurate diagnosis and to avoid the eventual poor outcome. With so few reports in the literature regarding spinal cord ischemia in the pediatric population, accurate diagnosis is often delayed until after irreversible events have already taken place. Our case report of anterior spinal artery distribution thoracic cord infarct following a minor trauma was diagnosed by axial thin-slice DWI MRI with an otherwise negative workup.
\end{abstract}

\section{Introduction}

Spinal cord ischemia is a rare occurrence in the adult and pediatric populations. Although cerebral ischemia is well understood, the natural history and prevalence of spinal cord ischemia are not well known given the paucity of cases in the literature $[1,2]$. It represents $\sim 6 \%$ of all acute myelopathies and can be difficult to differentiate from other forms of myelopathy [3]. It can present a diagnostic challenge to the emergency physician given that the clinical presentation varies depending on the involved vascular territory and can range from minor weakness to tetraplegia $[4,5]$. Some predisposing factors for spinal cord ischemia described in the literature include prior trauma, surgery, or congenital cardiovascular malformation. However, multiple cases were reported to occur in otherwise healthy

Ali Morshid

aimorshi@utmb.edu

1 Department of Diagnostic Radiology, The University of Texas Medical Branch, Galveston, TX, USA individuals or following minor trauma [6-8]. We present a case of anterior spinal artery territory infarction following minor trauma in a previously healthy 14 -year-old male who presented to the emergency department at our institution.

\section{Case presentation}

A 14-year-old right-handed male presented to our emergency department (ED) complaining of sudden onset stabbing back pain that started earlier that day at home. The patient reported an associated headache and denied any strenuous physical activity prior to the onset of pain. The patient recalled mild acute back pain that resolved spontaneously when he carried his cousin on his shoulders while at the pool one week prior to the current presentation.

Upon arrival to the ED, the patient reported numbness of bilateral lower extremities. His vital signs were within normal limits, he was alert, oriented, and reported improvement of his back pain after the administration of Ketorolac. Physical exam in the ED revealed localized tenderness over the upper back. Lower extremities' strength was within normal limits at the time of the initial 
examination. Initial imaging workup included thoracic and lumbar plain radiographs which were unremarkable. Laboratory workup revealed an elevated erythrocyte sedimentation rate value at 15 , white blood cell count of 12.2, and normal urine analysis. The initial differential diagnosis in the ED included traumatic injury to the spinal cord versus transverse myelitis.

The patient then started to demonstrate bilateral lower extremities weakness and was unable to ambulate independently. Pediatric neurology was consulted by the emergency physician and repeated physical exams revealed a sensory level at T5 with decreased pain and temperature sensation below that level. He also demonstrated a widebased gait with bent knees and an inability to stand independently. The International Standards for Neurological Classification of Spinal Cord Injury standardized examination was performed and revealed an incomplete T5 neurological level of injury with an American Spinal Injury Association impairment scale of $\mathrm{C}$.

The patient was admitted to the inpatient service and magnetic resonance imaging (MRI) of the cervical, thoracic, and lumbar spine was obtained. This MRI revealed a T2weighted signal hyperintensity involving the ventral thoracic cord at the midline spanning from T1 to T10 levels with no corresponding post-contrast enhancement or restricted diffusion on the provided sagittal diffusionweighted imaging sequence (DWI). The initial radiologic differential was formulated as inflammatory/infectious myelitis, autoimmune myelitis, acute demyelinating process, or transverse myelitis. Ischemic myelitis was considered a less likely possibility given the absence of diffusion restriction.

The differential diagnosis formulated by the pediatric neurologist included acute transverse myelitis, infectious myelitis, ischemic myelitis, neuromyelitis optica, or multiple sclerosis. The patient was then started on methylprednisolone and lumbar puncture was performed, which was inconclusive as the cerebrospinal fluid analysis returned negative for infection and CSF electrophoresis showed no oligoclonal bands. Acute flaccid myelitis was also considered as a less likely possibility given the sensory changes and the lack of preceding illness. Although the presumptive clinical diagnosis favored acute transverse myelitis by exclusion of other possibilities, the case was re-discussed with two neuroradiologists and both agreed that imaging findings were not characteristic of transverse myelitis and ischemic myelitis needed to be ruled out. This prompted vascular workup to rule out aortic dissection involving the artery of Adamkiewicz or radiculomedullary arteries, congenital cardiovascular malformations, and local shunting due to arteriovenous malformations. Computed tomography angiogram (CTA) of the chest was performed to rule out aortic dissection given the initial presentation of upper back pain and returned unremarkable for dissection but showed left T9 transverse process fracture. Cardiology service was consulted for evaluation of possible cardiovascular anomalies, and echocardiography was performed and was unremarkable. Enhanced and unenhanced MRI of the brain and orbits was then obtained and did not reveal any evidence of multiple sclerosis or neuromyelitis optica. The ophthalmologic exam did not reveal any evidence of optic disk edema or optic neuritis.

The patient then developed loss of deep tendon reflexes at the left knee and bilateral ankles approximately three days after admission and prompted repeat MRI of the thoracic spine. Only Sagittal thick slice sequences were performed initially as ischemic myelitis was not in the clinical differential diagnosis. Given the distribution of the T2/STIR hyperintense signal, repeat scan with thin-slice axial and sagittal DWI sequences was recommended which confirmed the diagnosis. Temporal evolution of the ischemic process might also have a role in producing more robust diffusion restriction signal. The repeat MRI again revealed a T2-weighted signal hyperintensity of the anterior aspect of the spinal cord spanning the level of T3-T10 with corresponding restricted diffusion on axial thin-slice DWI sequence and no post-contrast enhancement (Fig. 1). These findings were consistent with anterior spinal artery territory ischemic infarction. Marrow edema and post-contrast enhancement were observed at the left T9 transverse process that correlated with the non-displaced fracture on prior CTA of the chest (Fig. 2).

The patient started to demonstrate gradual clinical improvement after five days of high-dose steroids and physical therapy. Conventional spinal angiography was performed to rule out underlying vascular malformations or dissection with selective catheterization of the radiculomedullary branches and the artery of Adamkiewicz which was identified at the level of L1 (Fig. 3). Spinal angiogram was negative. The patient continued to demonstrate clinical improvement in gait and bilateral lower extremities weakness. He was discharged the next day on a tapering course of steroids with planned follow up with the pediatric neurology service.

\section{Discussion}

The anterior two-thirds of the spinal cord receive their arterial supply from the anterior spinal artery (ASA) and the posterior third from paired posterior spinal arteries [9]. The bilateral vertebral arteries contribute to the formation of the anterior spinal artery with additional contribution from the radiculomedullary branches of the extraspinal arteries $[10,11]$. In early fetal life, there are 31 pairs of radiculomedullary arteries, diminishing in number with individual 

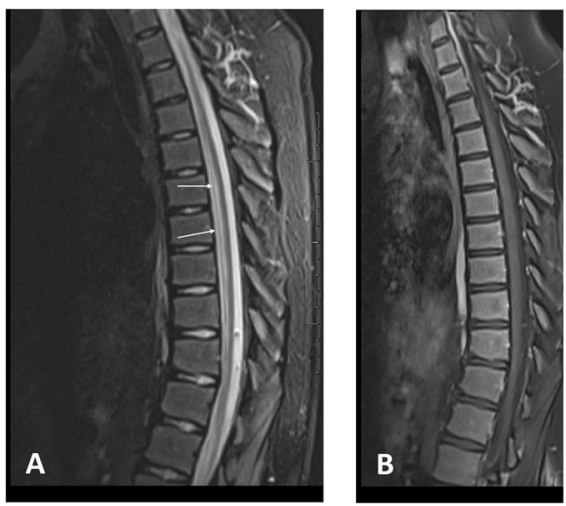

Fig. 1 Multi-sequence MRI of the thorathic spine. MRI of the thoracic spine demonstrates increased $\mathrm{T} 2$ signal intensity in the anterior spinal cord substance (arrows) on sagittal T2-weighted images (a), and no abnormal post-contrast enhancement on post-contrast sagittal
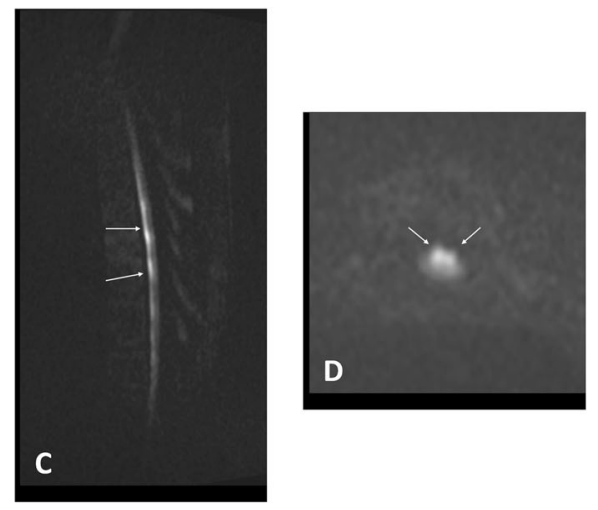

T1-weighted images (b). Sagittal DWI sequence (c) demonstrates restricted diffusion in the anterior spinal cord substance (arrows) which was better appreciated on axial thin-slice acquisition (d).
Fig. 2 Left T9 transverse process fracuture on MRI and CT. Axial post-contrast T1weighted image of the thoracic spine (a) demonstrates postcontrast enhancement of the left T9 transverse process (arrow). Axial (b), sagittal (c) and coronal (d) CT of the thoracic spine in the bone window confirms the presence of a nondisplaced minor fracture (arrow) of the left $\mathrm{T} 9$ transverse process.
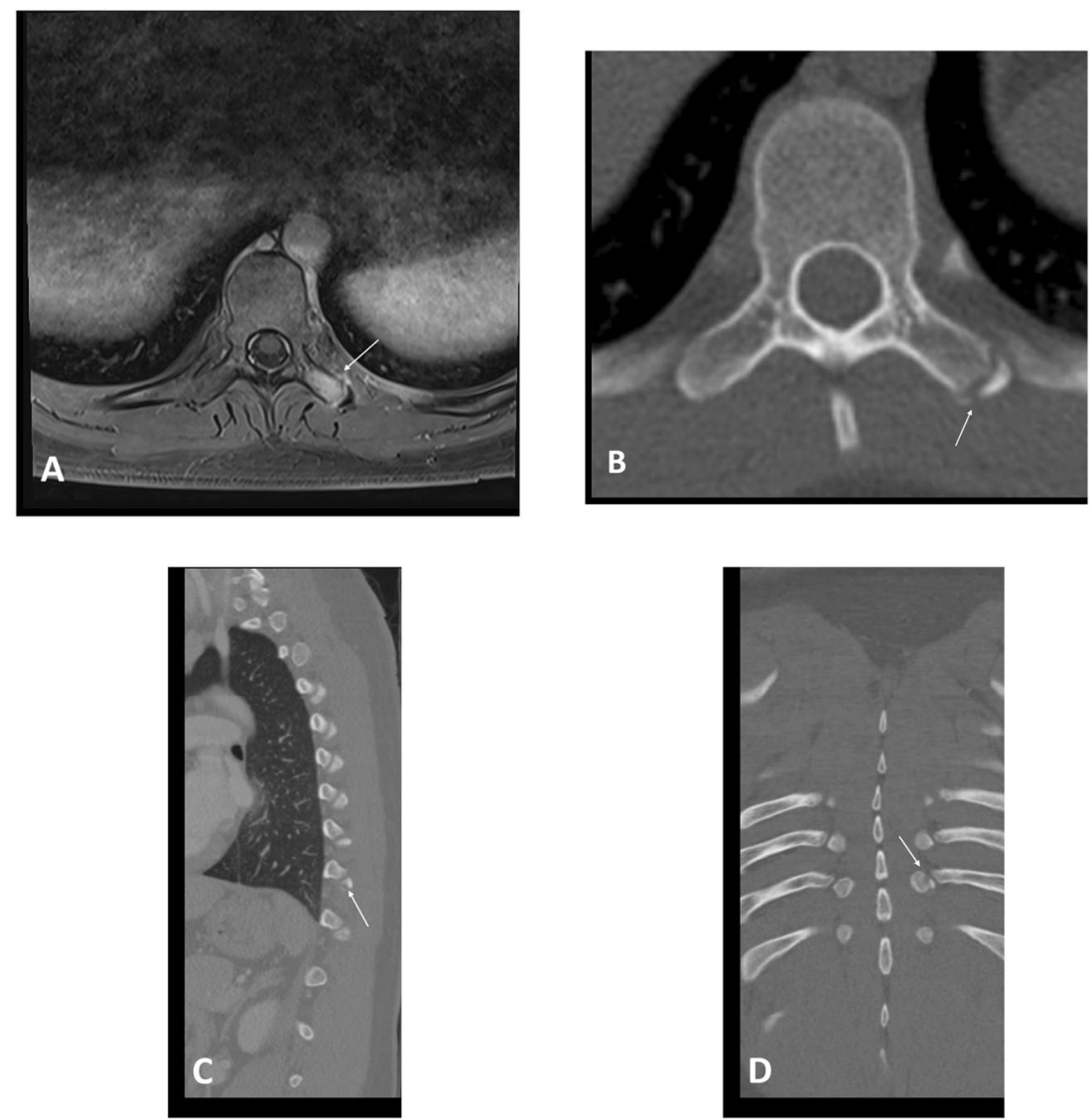

development varying from 2 to 14 and an average of six in adults [11]. In the thoracic and lumbar spine, the radicular arteries are supplied by intercostal and lumbar arteries that accompany the nerve root through the neural foramen and courses anterior to the ventral nerve root supplying the ASA with an average of two to three feeders in the thoracic spine and $0-1$ feeders in the lumbar spine [11]. The "hairpin loop" appearance is the term used to describe the morphology of the radiculomedullary arteries on angiogram as they join the ASA [11]. An additional contribution to the ASA is also identifiable in the thoracic and lumbar segments called the artery of Adamkiewicz [10]. It usually originates between $\mathrm{T} 9$ and $\mathrm{T} 12$, on the left side, and rarely from the lumbar or higher thoracic spine [10]. 
Fig. 3 Conventional spinal angiography with selective catheterization of the radiculomedullary branches.

Sample images of the radiculomedullary branch from the left T9 intercostal branch (a) and the artery of Adamkiewicz demonstrated at the level of L1 $(\mathbf{b}, \mathbf{c}$ and $\mathbf{d})$
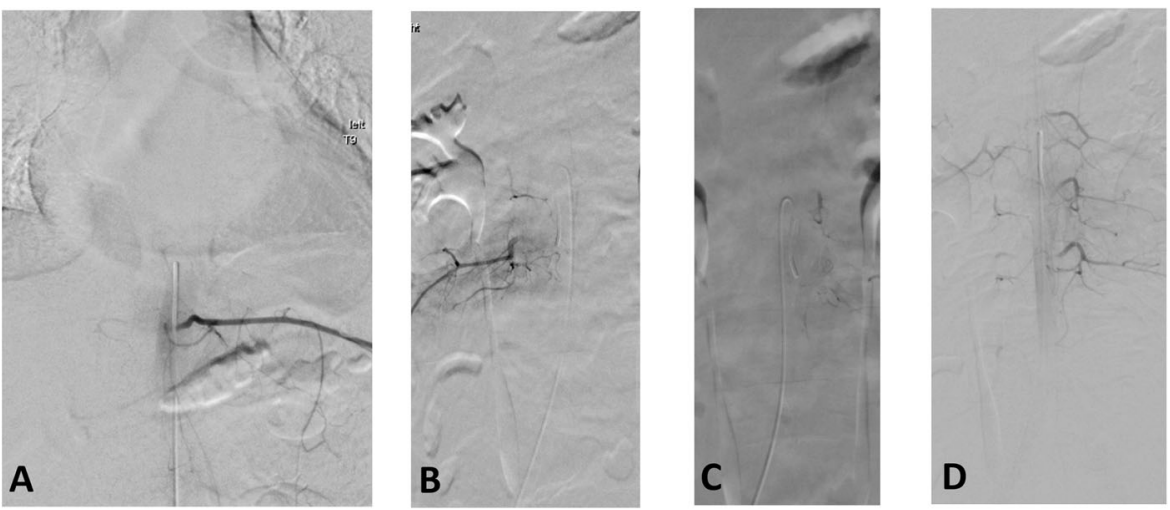

Given the rich vascularity of the spinal cord, infarctions are not common and estimated that they account for $\sim 1 \%$ of total adult strokes in the United States, not including cord infarctions secondary to aortic surgery complications $[3,12]$. Spinal cord infarction is even less common in pediatrics and incidence is not yet reported. Mechanism of pediatric spinal cord infarction is, the same as in adults, likely due to a decrease in blood supply secondary to hypotension or vascular occlusion as a sequela of traumatic or iatrogenic, thrombotic, or embolic disease. However, the underlying etiologies are different and not always recognized. Sladky and Rourke [13] found 9 of the examined 900 premature infants were having ischemic changes in the spinal cord on their post-mortem study and presented the hypothesis of immature autoregulation which was later supported by Singer et al.'s similar findings in three premature neonates [14]. Systemic hypotension due to cardiac arrest or in hypoxic-ischemic conditions can be a cause of cord infarction. Iatrogenic vascular injury has been reported in umbilical artery catheterization in a literature review by Sheikh et al. [2]. Aortic surgery as a cause of cord infarction is much less common than in adults but has been reported with the repair of coarctation of aorta and aortic dissection [15]. Other rare causes of vascular compromise such as embolization of esophageal varices, post lumbar puncture cerebellar herniation [16], and compression of cervicomedullary junction secondary to achondroplasia are also reported. Trauma is reported in the literature as a cause to be considered even when it is minor and not recalled by the child. The underlying mechanism is not well understood; Pang et al. [17, 18] attributed this to the theory that less pliable spinal cord against flexible vertebral column is likely the reason hyperflexion injuries can cause vascular spasm with subsequent vascular insult in the cord. Trauma can also cause vascular injury especially for radicular arteries or the artery of Adamkiewicz. Choi's et al theory about spinal cord infarction in the setting of thoracic or abdominal blunt trauma is that the small-caliber spinal arteries might get intimal tears that can eventually progress to dissection or thrombosis secondary to compression by hematoma or contusion [19]. Fibrocartilagenous embolization of nucleus pulposus has been a recognized cause of spinal cord infarction which could be post-traumatic or degenerative; the clue for diagnosis is intervertebral disc changes along with cord ischemia [8]. Often, even with extensive workup, etiology is not always identified or confirmed.

Nontraumatic thromboembolism to the spinal arteries is less common in children and likely secondary to vasculitis or thrombotic blood disorders like antiphospholipid syndrome, or sickle cell disease. Clinical presentation of spinal cord ischemia might mimic more common diagnostic possibilities like inflammatory, demyelinating, neoplastic processes, or post-traumatic contusions. MRI can suggest the diagnosis based on the pattern of cord involvement. DWI is the gold standard for the diagnosis of acute cerebral infarction, which demonstrates diffusion restriction. The same principle applies to the diagnosis of cord infarction and to differentiate it from other entities. The challenges with Spinal DWI are the presence of a small spinal canal and small cord diameter, and artifacts especially in the thoracic region secondary to respiration and CSF pulsation. Our recommendation is to obtain axial thin-slice DWI MRI for better resolution, as in our case, diffusion restriction was not called on the initial sagittal DWI but was evident on the axial thin slice sequence when repeated.

Our case report of anterior spinal artery distribution thoracic cord infarct was diagnosed by axial thin-slice DWI MRI with an otherwise negative workup. Given the acute, sudden onset upon lifting a child, the presence of a T9 transverse process fracture and the MRI findings, as well as a relatively normal spinal angiogram, it is postulated that the cord infarct was post-traumatic, with secondary injury to a radiculomedullary anterior spinal artery feeder or embolization to the thoracic portion of the anterior spinal artery. We believe that increased awareness of this entity and its etiology in the pediatric population can help clinicians and radiologists to consider it in difficult presentations. 


\section{Compliance with ethical standards}

Conflict of interest The authors declare that they have no conflict of interest.

Publisher's note Springer Nature remains neutral with regard to jurisdictional claims in published maps and institutional affiliations.

\section{References}

1. Cheshire WP, Santos CC, Massey EW, Howard JF. Spinal cord infarction: etiology and outcome. Neurology. 1996. https://doi. org/10.1212/WNL.47.2.321.

2. Sheikh A, Warren D, Childs AM, Russell J, Liddington M, Guruswamy V, et al. Paediatric spinal cord infarction-a review of the literature and two case reports. Child's Nerv Syst. 2017;33:671-6. https://doi.org/10.1007/s00381-016-3295-8.

3. Sandson TA, Friedman JH. Spinal cord infarction: report of 8 cases and review of the literature. Medicine. 1989. https://doi.org/ 10.1097/00005792-198909000-00003.

4. Vargas MI, Gariani J, Sztajzel R, Barnaure-Nachbar I, Delattre $\mathrm{BM}$, Lovblad KO, et al. Spinal cord ischemia: practical imaging tips, pearls, and pitfalls. Am J Neuroradiol. 2015;36:825-30. https://doi.org/10.3174/ajnr.A4118.

5. Novy J, Carruzzo A, Maeder P, Bogousslavsky J. Spinal cord ischemia: clinical and imaging patterns, pathogenesis, and outcomes in 27 patients. Arch Neurol. 2006. https://doi.org/10.1001/a rchneur.63.8.1113.

6. Nance JR, Golomb MR. Ischemic spinal cord infarction in children without vertebral fracture. Pediatr Neurol. 2007. pp. 209-216. https://doi.org/10.1016/j.pediatrneurol.2007.01.006. Accessed 20 Jul 2020.

7. Mathias E, Sethuraman U. Ischemic stroke of the spinal cord: a pediatric emergency in an otherwise healthy child. J Emerg Med. 2016;51:73-6. https://doi.org/10.1016/j.jemermed.2016.03.001.

8. Reisner A, Gary MF, Chern JJ, Grattan-Smith JD. Spinal cord infarction following minor trauma in children: fibrocartilaginous embolism as a putative cause: report of 3 cases. J Neurosurg: Pediatr. 2013;11:445-50. https://doi.org/10.3171/2013.1.PEDS12382.
9. Abecassis IJ, Osbun JW, Kim L. Classification and pathophysiology of spinal vascular malformations. Handbook of clinical neurology. Elsevier B.V. Amsterdam, Netherlands, 2017. pp. 135-143. https://doi.org/10.1016/B978-0-444-63640-9.00013-8. Accessed 23 Jul 2020.

10. Flores BC, Klinger DR, White JA, Batjer HH. Spinal vascular malformations: treatment strategies and outcome. Neurosurg Rev. 2017. 15-28. https://doi.org/10.1007/s10143-016-0713-z. Accessed 23 Jul 2020.

11. JAHAN R, VINUELA F. Vascular anatomy, pathophysiology, and classification of vascular malformations of the spinal cord. Semin Cerebrovasc Dis Stroke. 2002;2:186-200. https://doi.org/ 10.1053/scds.2002.127656.

12. Geldmacher DSBB. Vascular disease of the nervous system. In: Bradley WG, Daroff RB, Fenichel GMJJ (ed.) Vascular disease of the nervous system. Neurology in clinical practice. Butterworth Heinemann, London, 4th ed. 2004. pp.1313.

13. Sladky JT, Rorke LB. Perinatal hypoxic/ischemic spinal cord injury. Fetal Pediatr Pathol. 1986;6:87-101. https://doi.org/10. 3109/15513818609025927. Accessed 20 Jul 2020

14. Singer R, Joseph K, Gilai AN, Meyer S. Nontraumatic, acute neonatal paraplegia. J Pediatr Orthop. 1991;11:588-593. https:// doi.org/10.1097/01241398-199109000-00005. Accessed $20 \mathrm{Jul}$ 2020.

15. Brewer LA, Fosburg RG, Mulder GA, Verska JJ. Spinal cord complications following surgery for coarctation of the aorta. A study of 66 cases. J Thorac Cardiovasc Surg. 1972. https://doi. org/10.1016/S0022-5223(19)39832-0.

16. Michael Wieting J, Krach LE. Spinal cord injury rehabilitation in a pediatric achondroplastic patient: case report. Arch Phys Med Rehabil. 1994. https://doi.org/10.1016/0003-9993(94)90346-8.

17. Pang D, Wilberger JE. Spinal cord injury without radiographic abnormalities in children. J Neurosurg. 1982. https://doi.org/10. 3171/jns.1982.57.1.0114.

18. Pang D. Spinal cord injury without radiographic abnormality in children, 2 decades later. Neurosurgery. 2004. https://doi.org/10. 1227/01.NEU.0000143030.85589.E6.

19. Choi JU, Hoffman HJ, Hendrick EB, Humphreys RP, Keith WS. Traumatic infarction of the spinal cord in children. J Neurosurg. 1986;65:608-610. https://doi.org/10.3171/jns.1986.65.5.0608. Accessed 23 Jul 2020. 